\title{
A Role of Ginseng and Its Constituents in the Treatment of Central Nervous System Disorders
}

\author{
Natasya Trivena Rokot, ${ }^{1}$ Timothy Sean Kairupan, ${ }^{1,2}$ Kai-Chun Cheng, \\ Joshua Runtuwene, ${ }^{1,2}$ Nova Hellen Kapantow, ${ }^{2}$ Marie Amitani, ${ }^{1}$ Akinori Morinaga, ${ }^{1}$ \\ Haruka Amitani, ${ }^{1}$ Akihiro Asakawa, ${ }^{1}$ and Akio Inui ${ }^{1}$ \\ ${ }^{1}$ Department of Psychosomatic Internal Medicine, Kagoshima University Graduate School of Medical and Dental Sciences, \\ Kagoshima 890-8544, Japan \\ ${ }^{2}$ Faculty of Medicine, Sam Ratulangi University, Manado 95115, Indonesia
}

Correspondence should be addressed to Akio Inui; inui@m.kufm.kagoshima-u.ac.jp

Received 6 May 2016; Revised 20 July 2016; Accepted 26 July 2016

Academic Editor: Hongcai Shang

Copyright (C) 2016 Natasya Trivena Rokot et al. This is an open access article distributed under the Creative Commons Attribution License, which permits unrestricted use, distribution, and reproduction in any medium, provided the original work is properly cited.

\begin{abstract}
Ginseng, a perennial plant belonging to the Panax genus of the Araliaceae family, has been used in China, Korea, and Japan as a traditional herbal medicine for thousands of years. Ginseng is recorded to have exhibited a wide variety of beneficial pharmacological effects and has become a popular and worldwide known health supplement and drug. The protective effects of ginseng on central nervous system are discussed in this review. Ginseng species and ginsenosides and their intestinal metabolism and bioavailability are concisely introduced. The molecular mechanisms of the effects of ginseng on central nervous system, mainly focused on the neuroprotection properties of ginseng, memory, and learning enhanced properties, and the effects on neurodegenerative disorders are presented. Thus, ginseng and its constituents are of potential merits in the treatment of cerebral disorders.
\end{abstract}

\section{Introduction}

Ginseng has a medical history for thousands of years and become one of the most widely used traditional herbal medicines [1]. It belonged to the Panax genus of the Araliaceae family. The word Panax means "all heal" in Greek, which is based on the view that ginseng is powerful to heal any kind of disease. Ginseng is originated from the Chinese words "Jen Sheng," meaning "man-herb," because the shape in root of the plant resembles a humanoid form. The most extensively investigated ginsengs are Panax ginseng (Korean ginseng), Panax quinquefolius L. (American ginseng), and Panax notoginseng (Chinese ginseng) [2]. It has been documented that ginseng and its constituents exhibit a wide variety of beneficial pharmacological effects. Constituents of ginseng plant have been shown to produce adaptogenic, restorative, vasodilatory, immunomodulatory, anti-inflammatory, antioxidant, antiaging, anticancer, antifatigue, antidiabetic, antistress, and antidepressive effects in animals and humans [3-8].
Ginseng is also known to affect the nervous system, due to various effects that are beneficial to brain. Ginsenosides and other active constituents from ginseng are known to show neuroprotective properties and worked as cognitive performance and memory enhancer $[9,10]$. The purpose of this review is to discuss the effects of ginseng on central nervous system, mainly focused on the neuroprotection properties of ginseng, memory, and learning enhanced properties, and the effects on neurodegenerative disorders.

\section{Chemical Structure and Component}

The major active compounds in ginseng are triterpenoid glycosides, known also as the ginsenosides, contained in the roots, leaves, stems, flower buds, and berries. Ginsenosides are considered part of the defense mechanism in ginseng plants [11-16]. Identification and quantification of ginsenoside from ginseng plants have been established [17]. Ginsenosides consist of a 4-ring steroid backbone structure $[18,19]$. To date, more than 100 types of ginsenosides have been identified 
and isolated from the various parts of ginseng [9, 20-23]. Sugar types, quantities, and attachment positions, changeable carbon (C)-20 side chain, and stereoisomerism are the differentiating factors between each of ginsenosides [19, 24]. Generally, there are two major groups of ginsenosides: protopanaxadiols (PPD), including Rb1, Rb2, Rc, Rd, Rg3, Rh2, and Rh3; protopanaxatriols (PPT), including Re, Rf, Rg1, $\mathrm{Rg} 2$, and Rh1; and there is also the nonsteroidal saponin, oleanic acid group, which contained one ginsenoside, Ro [25]. Difference between two groups is the attached position of sugar moieties. In PPD group, the sugar moieties are attached to the $\mathrm{B}-\mathrm{OH}$ at $\mathrm{C}-3$ and/or $\mathrm{C}-20$, while in the PPT group they are attached to a-OH at C-6 and/or C-20 (Figure 1) [26, 27].

Besides the ginsenosides, other components are also found in ginseng, such as polysaccharides, flavonoids, volatile oils, and the recently identified nonsaponin compound called gintonin $[24,28]$.

\section{Bioavailability}

The oral bioavailability of ginsenosides is very poor. It cannot be easily absorbed by the intestines due to their hydrophilicity [29]. The absorption of ginsenosides in the intestinal mucosa is energy-dependent [30-32], and its availability of both intact ginsenosides and/or its metabolites from the intestines are very low [33-35]. Biotransformation of ginsenosides by microbiota in gut may form the deglycosylated products [36]. The deglycosylated products are more permeable and absorbable than ginsenosides [37]. However, the extensive biliary excretion through active transport causes the shortage of its biological half-life to result in a low systemic exposure level [36].

Some studies has been developed to overcome this problem, such as coadministration with adrenalin [38] or using lipid-based formulations $[39,40]$ and the suppression of $\mathrm{p}$ glycoprotein efflux system [30] that are proven to increase the oral bioavailability of ginsenosides.

\section{Effects on the Central Nervous System}

Ginseng and its constituents are known to have the beneficial effects on central nervous system (CNS) disorders including the cognitive performance, memory, and neurodegenerative diseases (Figure 2).

4.1. Neuroprotection. Many studies have identified the neuroprotective properties of ginseng and ginsenosides [41]. Ginsenosides Rb1 and Rg1 play a major role in neuroprotective effect. Rbl was shown to increase the neuron cell survival and improve neurite growth [42]. Rbl protects hippocampal neuron from the ischemic damage and also delays the neuronal death from transient forebrain ischemia [19]. Rg1 exerts a protective effect against the transient focal cerebral ischemic injury in rats with cerebral injury [43] and also protects AB25-35-induced cortical neuron apoptosis through the downregulation of nuclear factor-kappa $\mathrm{B}(\mathrm{NF}-\kappa \mathrm{B}) /$ nitric oxide (NO) signaling pathway [44]. An increase of membranes fluidity was observed in both Rbl and Rgl experiments [19], Rb1 enhanced the membrane fluidity of cortical cells
Ginsenoside Rb1

20(S)-protopanaxadiol

$\mathrm{R}_{1}=$ glu-glu

$\mathrm{R}_{2}=-\mathrm{H}$

$\mathrm{R}_{3}=$ glu-glu

Ginsenoside $\operatorname{Rg} 1$

20(S)-protopanaxatriol

$\mathrm{R}_{1}=-\mathrm{H}$

$\mathrm{R}_{2}=\mathrm{O}$-glu

$\mathrm{R}_{3}=$ glu

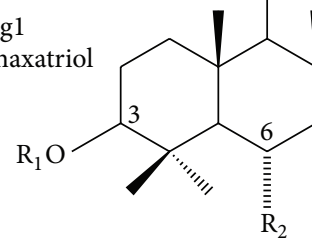

Figure 1: Structures of ginsenosides Rbl and Rgl. Based on the chemical structure, there are two major structural classes: the protopanaxadiol (PPD) and protopanaxatriol (PPT). Ginsenoside Rb1 is an example of PPD type and ginsenoside Rg1 is an example of PPT type.

in rats [45], and Rg1 increased the fluidity of synaptosomal membranes impaired by FeSO4-cysteine [46].

Otherwise, ginsenosides $\mathrm{Rd}$ and $\mathrm{Re}$ also have neuroprotective properties. Ginsenoside $\mathrm{Rd}$ is shown to ameliorate ischemic stroke-induced damage and prolong the neural cells' survival through several mechanism [47], such as phosphoinositide-3-kinase/AKT and phosphoextracellular signal-regulated protein kinase (ERK) 1/2 pathways [48], suppression of the NF- $\kappa \mathrm{B}$, transient receptor potential melastatin, acid sensing ion channels 1a [49], poly(ADP-ribose) polymerase-1 [50], protein tyrosine kinase activation, the upregulation of the endogenous antioxidant system, preservation of mitochondrial membrane potential, and reduction of cytochrome c-releasing and apoptosis-inducing factor [51-53]. Recent study suggested that Rd also promotes the neurites outgrowth, an important process for neuronal repair, of PC12 cells through upregulating GAP-43 expression via ERK- and ARK-dependent signaling [54]. Ginsenoside Re has been reported to decrease the mitochondrial swelling and prevent the reduction of $\mathrm{H}(+)$-ATPase activity in cerebral ischemia-reperfusion injury in rats [55].

4.2. Memory and Learning. Ginseng and its constituents have significant effects on memory and cognitive performances. Local administration of ginseng in brain-damaged rats showed a significant improvement in learning and memory [25]. Ginsenoside Rb1 has been reported to increase the uptake of choline in cerebral cholinergic nerve endings [56] and modulate acetylcholine (Ach) release and uptake [57], which related to learning process and memory. $\mathrm{Rbl}$ is also beneficial to cognitive impairment and hippocampus senescence [58]. Both Rb1 and Rg1 have been shown to improve the scopolamine-induced amnesia in rodents and also elevate the level of choline acetyltransferase (ChAT) in rodent brains $[59,60]$. Ginseng prevented the advance glycation end product- (AGE-) induced memory impairment by decreasing the expression of receptors for AGE (RAGEs) and $\kappa$-lightchainenhancer of activated $B$ cells $(N F-\kappa B)$ [61]. Chronic administration of ginsenoside to mice averts the memory lost and impairment $[62,63]$. Rg3 improved learning and 


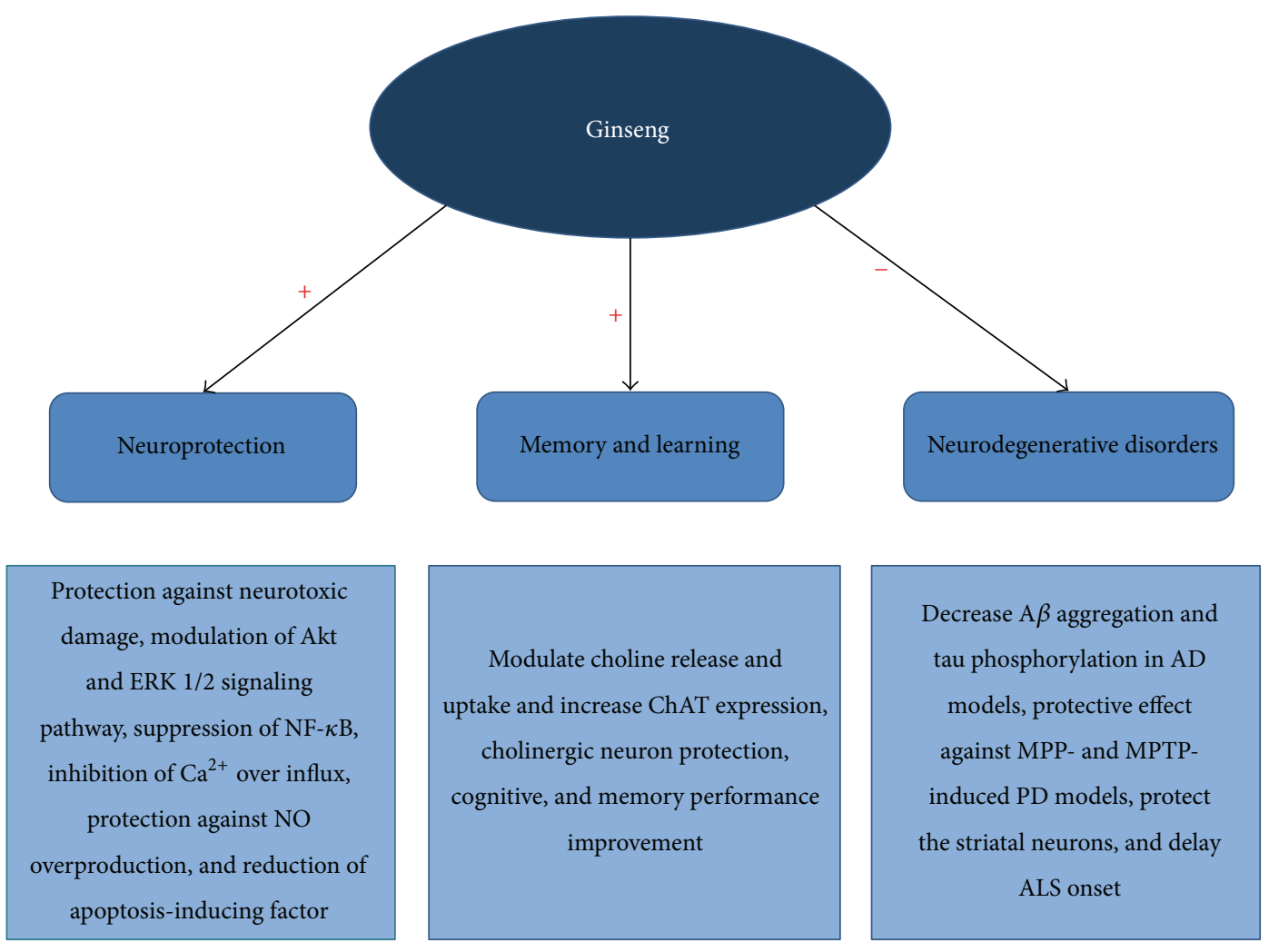

FIGURE 2: Multiple therapeutic targets of ginseng and its metabolites in central nervous system. ERK 1/2: extracellular-signal-regulated kinases 1 and 2, NF- $\kappa$ B: nuclear factor-kappa B, NO: nitric oxide, ChAT: choline acetyltransferase, A $\beta$ : $\beta$-amyloid, AD: Alzheimer's disease, MPTP: 1-methyl-4-phenyl-1,2,3,6-tetrahydropyridine, MPP: 1-methyl-4-phenylpyridium, PD: Parkinson's disease, and ALS: amyotrophic lateral sclerosis.

memory impairments in lipopolysaccharide-induced cognitive impairment [64]. Rg3 and $\mathrm{Rg} 5 / \mathrm{Rg} 1$ administration were also shown to enhance memory in scopolamine or ethanolinduce memory dysfunction in mice [65].

Other compounds like gintonin also possessed the ability to improve cognitive functions. Systemic administration of gintonin showed an improvement in contextual memory formation at molecular level up to behavioral level in experimental mice [66].

4.3. Neurodegenerative Diseases. The merits of ginseng and ginsenosides also included the neurodegenerative diseases (Table 1). Neurodegenerative diseases are associated with progressive loss of structure or neuron function, with loss of cognitive function and motor disabilities. Neurodegenerative diseases include Alzheimer's disease (AD), Parkinson's disease (PD), Huntington's disease (HD), and amyotrophic lateral sclerosis (ALS).

4.3.1. Alzheimer's Disease. AD accounts for more than 60$70 \%$ of dementia, a general term for a memory disorder, including the loss of memory, and other intellectual abilities, which are great enough to interfere with the person's daily life. One of the major pathological features of AD is the presence of $\beta$-amyloid $(\mathrm{A} \beta)$ around arterioles or capillaries wall in the brain [81]. Administration of ginseng and its constituents may inhibit $\mathrm{A} \beta$ aggregation in cultured neurons. Ginsenoside Rg1 administration showed a significant reduction in cerebral $\mathrm{A} \beta$ in aged transgenic AD mice, with improved spatial learning abilities and memory [67]. Gintonin also is shown to improve $\mathrm{AD}$, by attenuating the deposition of amyloid plaque, and memory impairment in AD mouse [68]. A $\beta$ peptides resulted from the amyloid precursor protein (APP), cleaved by beta secretase (BACE1) and gamma secretase. Several studies have showed that ginseng and ginsenosides have the abilities to enhance the nonamyloidogenic processing of APP by increasing $\alpha$ secretase activities and decrease the amyloidogenic processing by decreasing BACE1 $[68,69]$.

Hyperphosphorylated tau protein is also known to cause AD. Hyperphosphorylation of tau protein will lead to the accumulation of neurofibrillary tangles inside nerve cell bodies. This event will lead to an interference of the cellular transport process in brain [82]. Total ginsenosides extracted from stems and leaves of Panax ginseng are shown to inhibit tau hyperphosphorylation by enhancing the phosphatase activity of purified calcineurin in SY5Y cells [70]. Ginsenosides Rd and $\mathrm{Rbl}$ are also shown to reduce hyperphosphorylated tau by enhancing phosphatase 2A level (PP2A) [71]. Rg1 was shown to reverse the memory impairments by decreasing hyperphosphorylated tau and suppressing $\mathrm{A} \beta$ formation in rat brain [83].

A decrease of cholinergic neurons in brain is associated with $\mathrm{AD}$. Ginsenoside Rg5 has been shown to improve 
TABLE 1: Effects of ginseng and its metabolites in neurodegenerative disorder. AD: Alzheimer's disease, PD: Parkinson's disease, HD: Huntington's disease, ALS: amyotrophic lateral sclerosis, and A $\beta$ : $\beta$-amyloid.

\begin{tabular}{lccc}
\hline $\begin{array}{l}\text { Neurodegenerative } \\
\text { disorder }\end{array}$ & Active compound & Research type & Mechanism \\
\hline AD & $\begin{array}{c}\text { Rb1, Rg1, Rg5, Rd, Re, gintonin, } \\
\text { and ginseng extract panaxynol }\end{array}$ & Animal model and cell culture & $\begin{array}{c}\text { Decrease A } \beta \text { production and } \\
\text { aggregation, increase A } \beta \text { clearance, } \\
\text { decrease of tau hyperphosphorylation, } \\
\text { and improve cholinergic function }\end{array}$ \\
\hline [67-73] & $\begin{array}{c}\text { Protection against neurotoxic damage } \\
\text { and inhibition of a-synuclein } \\
\text { aggregation }\end{array}$ \\
\hline [D & Rb, Rgl, and ginseng extract G115 & $\begin{array}{c}\text { Animal model and } \\
\text { cell culture }\end{array}$ & $\begin{array}{c}\text { Protection against neurotoxic damage } \\
\text { and inhibition of Ca }{ }^{2+} \text { signaling }\end{array}$ \\
\hline ALS & Rb1, Rc, and Rg5 & Animal model & Unknown \\
\hline
\end{tabular}

cognitive dysfunction and neuroinflammation and modulate both AChE and ChAT activity in brain cortex [72]. Other ginsenosides, Re and Rd, are also shown to enhance ChAT and vascular acetylcholine transporter (VAChT) to increase the Ach level in neuro-21 cells [73].

4.3.2. Parkinson's Disease. PD is a neurodegenerative disorder affecting mainly the motor system, as a result of the death of dopaminergic neurons in substantia nigra (SN). Administration of 1-methyl-4-phenyl-1,2,3,6-tetrahydropyridine (MPTP) or its metabolite 1-methyl-4-phenylpyridium $\left(\mathrm{MPP}^{+}\right)$has been used to induce PD models in various animal studies. MPTP and $\mathrm{MPP}^{+}$destroy dopaminergic neurons in substantia nigra, which causes PD symptoms. Treatment of ginseng extracts produced neuroprotective effect in $\mathrm{PD}$ mouse model $[74,84]$. Rbl and Rgl are shown to inhibit the decrease of neurite length or numbers in MPP-treated primary dopaminergic cultures [75]. Rg1 has been reported to reduce MPTP-induced substantia nigra neuronal loss in C57/BL6 mice [74, 76]. Oral intake of ginseng extract, G115, significantly prevented tyrosine hydroxylase-positive cell loss in substantia nigra and attenuated the locomotor dysfunction in MPTP treated rodents [77]. G115 has also been shown to reduce dopaminergic cell loss in $\beta$-sitosterol $\beta$-d-glucoside fed rats (BSSG rat model of Parkinson's disease) [78].

4.3.3. Other Neurodegenerative Diseases. In addition to the effects on $\mathrm{AD}$ and $\mathrm{PD}$, ginseng and its constituent also showed similar influence to other neurodegenerative diseases, including Huntington's diseases and amyotrophic lateral sclerosis. Ginsenosides have been shown to protect the striatal neurons in cellular models of HD [79]. Moreover, ginseng extract also delayed the ALS onset in B6SJL-TgN(SOD1-G93A)1Gur transgenic mice [80]. But further studies are still required to determine the effectiveness and elucidate their mechanisms of action of ginseng or its constituents in other neurodegenerative diseases.

\section{Conclusions and Future Perspective}

Ginseng has been used for thousands of years as traditional medicine. The results reviewed above from cell culture systems, animal studies, and human studies suggest that ginseng and its constituents are effective to produce the beneficial effects on CNS, including neuroprotection, cognitive, and memory performance enhancement. However, the active compounds, ginsenosides and gintonin, which influenced the CNS have not been fully elucidated. Further studies are still necessary to unravel the mechanisms of action, detailed pharmacokinetics and toxicity, standardization of each ginseng preparation, and therapeutic doses in animals and humans. Additionally, the clinical trials are still required to confirm the effectiveness of ginseng and its constituents in modulating these neurodegenerative diseases. Overall, ginseng and its constituents are merits in the treatment of cerebral disorders.

\section{Competing Interests}

The authors declare that there are no competing interests regarding the publication of this paper.

\section{References}

[1] Y.-Z. Xiang, H.-C. Shang, X.-M. Gao, and B.-L. Zhang, "A comparison of the ancient use of ginseng in traditional Chinese medicine with modern pharmacological experiments and clinical trials," Phytotherapy Research, vol. 22, no. 7, pp. 851-858, 2008.

[2] G.-H. Lu, Q. Zhou, S.-Q. Sun, K. S.-Y. Leung, H. Zhang, and Z.-Z. Zhao, "Differentiation of Asian ginseng, American ginseng and Notoginseng by Fourier transform infrared spectroscopy combined with two-dimensional correlation infrared spectroscopy," Journal of Molecular Structure, vol. 883-884, no. 1-3, pp. 91-98, 2008.

[3] Y. Cheng, L.-H. Shen, and J.-T. Zhang, "Anti-amnestic and antiaging effects of ginsenoside Rgl and Rbl and its mechanism of action," Acta Pharmacologica Sinica, vol. 26, no. 2, pp. 143-149, 2005.

[4] H. R. Shin, J. Y. Kim, T. K. Yun, G. Morgan, and H. Vainio, "The cancer-preventive potential of Panax ginseng: a review of human and experimental evidence," Cancer Causes and Control, vol. 11, no. 6, pp. 565-576, 2000.

[5] M.-K. Choo, E.-K. Park, M. J. Han, and D.-H. Kim, "Antiallergic activity of ginseng and its ginsenosides," Planta Medica, vol. 69, no. 6, pp. 518-522, 2003.

[6] D. Y. Kim and W. M. Yang, "Panax ginseng ameliorates airway inflammation in an ovalbumin-sensitized mouse allergic 
asthma model," Journal of Ethnopharmacology, vol. 136, no. 1, pp. 230-235, 2011.

[7] K.-C. Cheng, Y.-X. Li, and J.-T. Cheng, "The use of herbal medicine in cancer-related anorexia/ cachexia treatment around the world," Current Pharmaceutical Design, vol. 18, no. 31, pp. 4819-4826, 2012.

[8] T. Liu, I. Liu, and J. Cheng, "Improvement of insulin resistance by panax ginseng in fructose-rich chow-fed rats," Hormone and Metabolic Research, vol. 37, no. 3, pp. 146-151, 2005.

[9] L. P. Christensen, "Chapter 1 ginsenosides. Chemistry, biosynthesis, analysis, and potential health effects," Advances in Food and Nutrition Research, vol. 55, pp. 1-99, 2008.

[10] K. Radad, R. Moldzio, and W.-D. Rausch, "Ginsenosides and their CNS Targets," CNS Neuroscience and Therapeutics, vol. 17, no. 6, pp. 761-768, 2011.

[11] W. S. Sung and D. G. Lee, "In vitro candidacidal action of Korean red ginseng saponins against Candida albicans," Biological and Pharmaceutical Bulletin, vol. 31, no. 1, pp. 139-142, 2008.

[12] D.-W. Choi, J. Jung, Y. I. Ha et al., "Analysis of transcripts in methyl jasmonate-treated ginseng hairy roots to identify genes involved in the biosynthesis of ginsenosides and other secondary metabolites," Plant Cell Reports, vol. 23, no. 8, pp. 557-566, 2005.

[13] D. R. Katerere, A. I. Gray, R. J. Nash, and R. D. Waigh, "Antimicrobial activity of pentacyclic triterpenes isolated from African Combretaceae," Phytochemistry, vol. 63, no. 1, pp. 81-88, 2003.

[14] U. V. Mallavadhani, A. Mahapatra, S. S. Raja, and C. Manjula, "Antifeedant activity of some pentacyclic triterpene acids and their fatty acid ester analogues," Journal of Agricultural and Food Chemistry, vol. 51, no. 7, pp. 1952-1955, 2003.

[15] R. W. Nicol, J. A. Traquair, and M. A. Bernards, "Ginsenosides as host resistance factors in American ginseng (Panax quinquefolius)," Canadian Journal of Botany, vol. 80, no. 5, pp. 557-562, 2002.

[16] R. A. Creelman and J. E. Mullet, "Biosynthesis and action of jasmonates in plants," Annual Review of Plant Biology, vol. 48, pp. 355-381, 1997.

[17] N. Fuzzati, "Analysis methods of ginsenosides," Journal of Chromatography B, vol. 812, no. 1-2, pp. 119-133, 2004.

[18] A. Wang, C.-Z. Wang, J.-A. Wu, J. Osinski, and C.-S. Yuan, "Determination of major ginsenosides in Panax quinquefolius (American ginseng) using high-performance liquid chromatography," Phytochemical Analysis, vol. 16, no. 4, pp. 272-277, 2005.

[19] A. S. Attele, J. A. Wu, and C.-S. Yuan, "Ginseng pharmacology: multiple constituents and multiple actions," Biochemical Pharmacology, vol. 58, no. 11, pp. 1685-1693, 1999.

[20] M. Yoshikawa, S. Sugimoto, S. Nakamura, and H. Matsuda, "Medicinal flowers. XI. Structures of new dammarane-type triterpene diglycosides with hydroperoxide group from flower buds of Panax ginseng," Chemical and Pharmaceutical Bulletin, vol. 55, no. 4, pp. 571-576, 2007.

[21] E. Tachikawa, K. Kudo, K. Harada et al., "Effects of ginseng saponins on responses induced by various receptor stimuli," European Journal of Pharmacology, vol. 369, no. 1, pp. 23-32, 1999.

[22] M. Yoshikawa, T. Murakami, K. Yashiro et al., "Bioactive saponins and glycosides. XI. Structures of new dammaranetype triterpene oligoglycosides, quinquenosides I, II, III, IV, and $\mathrm{V}$, from American ginseng, the roots of Panax quinquefolium L.," Chemical and Pharmaceutical Bulletin, vol. 46, no. 4, pp. 647-654, 1998.
[23] B.-K. Shin, S. W. Kwon, and J. H. Park, "Chemical diversity of ginseng saponins from Panax ginseng," Journal of Ginseng Research, vol. 39, no. 4, pp. 287-298, 2015.

[24] L. Jia and Y. Zhao, "Current evaluation of the millennium phytomedicine-ginseng (I): etymology, pharmacognosy, phytochemistry, market and regulations," Current Medicinal Chemistry, vol. 16, no. 19, pp. 2475-2484, 2009.

[25] D. O. Kennedy and A. B. Scholey, "Ginseng: potential for the enhancement of cognitive performance and mood," Pharmacology Biochemistry and Behavior, vol. 75, no. 3, pp. 687-700, 2003.

[26] C. Qu, Y. Bai, X. Jin et al., "Study on ginsenosides in different parts and ages of Panax quinquefolius L.," Food Chemistry, vol. 115, no. 1, pp. 340-346, 2009.

[27] H. Matsuura, R. Kasai, O. Tanaka, Y. Saruwatari, K. Kunihiro, and T. Fuwa, "Further studies on dammarane-saponins of ginseng roots," Chemical and Pharmaceutical Bulletin, vol. 32, no. 3, pp. 1188-1192, 1984.

[28] M. K. Pyo, S.-H. Choi, S. H. Hwang et al., "Novel glycolipoproteins from ginseng," Journal of Ginseng Research, vol. 35, no. 1, pp. 92-103, 2011.

[29] D.-H. Kim, "Chemical diversity of Panax ginseng, Panax quinquifolium, and Panax notoginseng," Journal of Ginseng Research, vol. 36, no. 1, pp. 1-15, 2012.

[30] H.-T. Xie, G.-J. Wang, M. Chen et al., "Uptake and metabolism of ginsenoside Rh2 and its aglycon protopanaxadiol by Caco-2 cells," Biological and Pharmaceutical Bulletin, vol. 28, no. 2, pp. 383-386, 2005.

[31] M. Han and X.-L. Fang, "Difference in oral absorption of ginsenoside Rg1 between in vitro and in vivo models," Acta Pharmacologica Sinica, vol. 27, no. 4, pp. 499-505, 2006.

[32] M. Han, X. Sha, Y. Wu, and X. Fang, "Oral absorption of ginsenoside Rb1 using in vitro and in vivo models," Planta Medica, vol. 72, no. 5, pp. 398-404, 2006.

[33] Q. F. Xu, X. L. Fang, and D. F. Chen, "Pharmacokinetics and bioavailability of ginsenoside Rb1 and Rgl from Panax notoginseng in rats," Journal of Ethnopharmacology, vol. 84, no. 2-3, pp. 187-192, 2003.

[34] Y. Takino, "Studies on the pharmacodynamics of ginsenosideRg1, -Rb1 and -Rb2 in rats," Yakugaku Zasshi, vol. 114, no. 8, pp. 550-564, 1994.

[35] M. Karikura, T. Miyase, H. Tanizawa, Y. Takino, T. Taniyama, and T. Hayashi, "Studies on absorption, distribution, excretion and metabolism of Ginseng saponins. V. The decomposition products of ginsenoside b2 in the large intestine of rats," Chemical and Pharmaceutical Bulletin, vol. 38, no. 10, pp. 28592861, 1990.

[36] H. Liu, J. Yang, F. Du et al., "Absorption and disposition of ginsenosides after oral administration of Panax notoginseng extract to rats," Drug Metabolism and Disposition, vol. 37, no. 12, pp. 2290-2298, 2009.

[37] H. Hasegawa and M. Uchiyama, "Antimetastatic efficacy of orally administered ginsenoside Rb1 in dependence on intestinal bacterial hydrolyzing potential and significance of treatment with an active bacterial metabolite," Planta Medica, vol. 64, no. 8, pp. 696-700, 1998.

[38] J. Xiong, M. Sun, J. Guo et al., "Enhancement by adrenaline of ginsenoside Rgl transport in Caco-2 cells and oral absorption in rats," Journal of Pharmacy and Pharmacology, vol. 61, no. 3, pp. 347-352, 2009.

[39] J. Xiong, J. Guo, L. Huang, B. Meng, and Q. Ping, "The use of lipid-based formulations to increase the oral bioavailability of 
panax notoginseng saponins following a single oral gavage to rats," Drug Development and Industrial Pharmacy, vol. 34, no. 1, pp. 65-72, 2008.

[40] M. Han, S. Fu, J.-Q. Gao, and X.-L. Fang, "Evaluation of intestinal absorption of ginsenoside Rgl incorporated in microemulison using parallel artificial membrane permeability assay," Biological and Pharmaceutical Bulletin, vol. 32, no. 6, pp. 10691074, 2009.

[41] W.-D. Rausch, S. Liu, G. Gille, and K. Radad, "Neuroprotective effects of ginsenosides," Acta Neurobiologiae Experimentalis, vol. 66, no. 4, pp. 369-375, 2006.

[42] J. Liu, J. He, L. Huang, L. Dou, S. Wu, and Q. L. Yuan, "Neuroprotective effects of ginsenoside Rb1 on hippocampal neuronal injury and neurite outgrowth," Neural Regeneration Research, vol. 9, no. 9, pp. 943-950, 2014.

[43] M. Lin, W. Sun, W. Gong, Y. Ding, Y. Zhuang, and Q. Hou, "Ginsenoside Rg1 protects against transient focal cerebral ischemic injury and suppresses its systemic metabolic changes in cerabral injury rats," Acta Pharmaceutica Sinica B, vol. 5, no. 3, pp. 277284, 2015.

[44] J. Wu, H. Yang, Q. Zhao, X. Zhang, and Y. Lou, "Ginsenoside Rg1 exerts a protective effect against $\mathrm{A} \beta 25$-35-induced toxicity in primary cultured rat cortical neurons through the NF- $\kappa \mathrm{B} / \mathrm{NO}$ pathway," International Journal of Molecular Medicine, vol. 37, no. 3, pp. 781-788, 2016.

[45] J. Q. Li and J. T. Zhang, "Effects of age and Ginsenoside RG1 on membrane fluidity of cortical cells in rats," Yaoxue Xuebao, vol. 32, no. 1, pp. 23-27, 1997.

[46] X. Y. Jiang, J. T. Zhang, and C. Z. Shi, "Mechanism of action of ginsenoside $\mathrm{Rb} 1$ in decreasing intracellular $\mathrm{Ca} 2+$," Yaoxue Xuebao, vol. 31, no. 5, pp. 321-326, 1996.

[47] S. F. Nabavi, A. Sureda, S. Habtemariam, and S. M. Nabavi, "Ginsenoside Rd and ischemic stroke; a short review of literatures," Journal of Ginseng Research, vol. 39, no. 4, pp. 299-303, 2015.

[48] X. Zhang, M. Shi, M. Bjørås et al., "Ginsenoside Rd promotes glutamate clearance by up-regulating glial glutamate transporter GLT-1 via PI3K/AKT and ERK1/2 pathways," Frontiers in Pharmacology, vol. 4, pp. 152-158, 2013.

[49] Y. Zhang, L. Zhou, X. Zhang, J. Bai, M. Shi, and G. Zhao, "Ginsenoside-Rd attenuates TRPM7 and ASICla but promotes ASIC2a expression in rats after focal cerebral ischemia," Neurological Sciences, vol. 33, no. 5, pp. 1125-1131, 2012.

[50] G. Hu, Z. Wu, F. Yang et al., "Ginsenoside Rd blocks AIF mitochondrio-nuclear translocation and NF- $\kappa$ B nuclear accumulation by inhibiting poly(ADP-ribose) polymerase-1 after focal cerebral ischemia in rats," Neurological Sciences, vol. 34, no. 12, pp. 2101-2106, 2013.

[51] R. Ye, N. Li, J. Han et al., "Neuroprotective effects of ginsenoside $\mathrm{Rd}$ against oxygen-glucose deprivation in cultured hippocampal neurons," Neuroscience Research, vol. 64, no. 3, pp. 306-310, 2009.

[52] R. Ye, Q. Yang, X. Kong et al., "Ginsenoside Rd attenuates early oxidative damage and sequential inflammatory response after transient focal ischemia in rats," Neurochemistry International, vol. 58, no. 3, pp. 391-398, 2011.

[53] R. Ye, X. Kong, Q. Yang, Y. Zhang, J. Han, and G. Zhao, "Ginsenoside Rd attenuates redox imbalance and improves stroke outcome after focal cerebral ischemia in aged mice," Neuropharmacology, vol. 61, no. 4, pp. 815-824, 2011.

[54] S.-D. Wu, F. Xia, X.-M. Lin et al., "Ginsenoside-Rd promotes neurite outgrowth of PC12 cells through MAPK/ERK- and PI3K/AKT-dependent pathways," International Journal of Molecular Sciences, vol. 17, no. 2, p. 177, 2016.

[55] L.-M. Chen, X.-M. Zhou, Y.-L. Cao, and W.-X. Hu, "Neuroprotection of ginsenoside Re in cerebral ischemia-reperfusion injury in rats," Journal of Asian Natural Products Research, vol. 10, no. 5, pp. 439-445, 2008.

[56] C. G. Benishin, "Actions of ginsenoside Rb1 on choline uptake in central cholinergic nerve endings," Neurochemistry International, vol. 21, no. 1, pp. 1-5, 1992.

[57] C. G. Benishin, R. Lee, L. C. H. Wang, and H. J. Liu, "Effects of ginsenoside Rb1 on central cholinergic metabolism," Pharmacology, vol. 42, no. 4, pp. 223-229, 1991.

[58] J. Zhu, X. Mu, J. Zeng et al., "Ginsenoside Rgl prevents cognitive impairment and hippocampus senescence in a rat model of Dgalactose-induced aging," PLoS ONE, vol. 9, no. 6, Article ID e101291, 2014.

[59] J. T. Zhang, Z. W. Qu, Y. Liu, and H. L. Deng, "Preliminary study on antiamnestic mechanism of ginsenoside Rg1 and Rbl," Chinese Medical Journal, vol. 103, no. 11, pp. 932-938, 1990.

[60] K. N. Salim, B. S. McEwen, and H. M. Chao, "Ginsenoside Rb1 regulates ChAT, NGF and trkA mRNA expression in the rat brain," Molecular Brain Research, vol. 47, no. 1-2, pp. 177-182, 1997.

[61] X. Tan, J. Gu, B. Zhao et al., "Ginseng improves cognitive deficit via the RAGE/NF- $\kappa$ B pathway in advanced glycation end product-induced rats," Journal of Ginseng Research, vol. 39, no. 2, pp. 116-124, 2015.

[62] H. Zhao, Q. Li, X. Pei et al., "Long-term ginsenoside administration prevents memory impairment in aged C57BL/6J mice by up-regulating the synaptic plasticity-related proteins in hippocampus," Behavioural Brain Research, vol. 201, no. 2, pp. 311-317, 2009.

[63] H. Zhao, Q. Li, Z. Zhang, X. Pei, J. Wang, and Y. Li, "Longterm ginsenoside consumption prevents memory loss in aged SAMP8 mice by decreasing oxidative stress and up-regulating the plasticity-related proteins in hippocampus," Brain Research, vol. 1256, pp. 111-122, 2009.

[64] B. Lee, B. Sur, J. Park et al., "Ginsenoside Rg3 alleviates lipopolysaccharide-induced learning and memory impairments by antiinflammatory activity in rats," Biomolecules and Therapeutics, vol. 21, no. 5, pp. 381-390, 2013.

[65] H. Y. Bao, J. Zhang, S. J. Yeo et al., "Memory enhancing and neuroprotective effects of selected ginsenosides," Archives of Pharmacal Research, vol. 28, no. 3, pp. 335-342, 2005.

[66] S. Kim, M.-S. Kim, K. Park et al., "Hippocampus-dependent cognitive enhancement induced by systemic gintonin administration," Journal of Ginseng Research, vol. 40, no. 1, pp. 55-61, 2016.

[67] F. Fang, X. Chen, T. Huang, L.-F. Lue, J. S. Luddy, and S. S. Yan, "Multi-faced neuroprotective effects of Ginsenoside Rg1 in an Alzheimer mouse model," Biochimica et Biophysica Acta (BBA)-Molecular Basis of Disease, vol. 1822, no. 2, pp. 286-292, 2012.

[68] S. H. Hwang, E.-J. Shin, T.-J. Shin et al., "Gintonin, a ginsengderived lysophosphatidic acid receptor ligand, attenuates alzheimer's disease-related neuropathies: involvement of nonamyloidogenic processing," Journal of Alzheimer's Disease, vol. 31, no. 1, pp. 207-223, 2012.

[69] V. Karpagam, N. Sathishkumar, S. Sathiyamoorthy et al., "Identification of BACE1 inhibitors from Panax ginseng saponins-An Insilco approach," Computers in Biology and Medicine, vol. 43, no. 8, pp. 1037-1044, 2013. 
[70] L.-H. Tu, J. Ma, H.-P. Liu, R.-R. Wang, and J. Luo, “The neuroprotective effects of ginsenosides on calcineurin activity and tau phosphorylation in SY5Y cells," Cellular and Molecular Neurobiology, vol. 29, no. 8, pp. 1257-1264, 2009.

[71] L. Li, J. Liu, X. Yan et al., "Protective effects of ginsenoside Rd against okadaic acid-induced neurotoxicity in vivo and in vitro," Journal of Ethnopharmacology, vol. 138, no. 1, pp. 135-141, 2011.

[72] S. Chu, J. Gu, L. Feng et al., "Ginsenoside Rg5 improves cognitive dysfunction and beta-amyloid deposition in STZ-induced memory impaired rats via attenuating neuroinflammatory responses," International Immunopharmacology, vol. 19, no. 2, pp. 317-326, 2014.

[73] M. S. Kim, J. M. Yu, H. J. Kim et al., "Ginsenoside Re and Rd enhance the expression of cholinergic markers and neuronal differentiation in Neuro-2a cells," Biological and Pharmaceutical Bulletin, vol. 37, no. 5, pp. 826-833, 2014.

[74] X.-C. Chen, Y.-C. Zhou, Y. Chen, Y.-G. Zhu, F. Fang, and L.M. Chen, "Ginsenoside Rg1 reduces MPTP-induced substantia nigra neuron loss by suppressing oxidative stress," Acta Pharmacologica Sinica, vol. 26, no. 1, pp. 56-62, 2005.

[75] K. Radad, G. Gille, R. Moldzio, H. Saito, K. Ishige, and W.D. Rausch, "Ginsenosides Rbl and Rg1 effects on survival and neurite growth of MPP +-affected mesencephalic dopaminergic cells," Journal of Neural Transmission, vol. 111, no. 1, pp. 37-45, 2004.

[76] X.-C. Chen, Y. Chen, Y.-G. Zhu, F. Fang, and L.-M. Chen, "Protective effect of ginsenoside Rg1 against MPTP-induced apoptosis in mouse substantia nigra neurons," Acta Pharmacologica Sinica, vol. 23, no. 9, pp. 829-834, 2002.

[77] J. Van Kampen, H. Robertson, T. Hagg, and R. Drobitch, "Neuroprotective actions of the ginseng extract G115 in two rodent models of Parkinson's disease," Experimental Neurology, vol. 184, no. 1, pp. 521-529, 2003.

[78] J. M. Van Kampen, D. B. Baranowski, C. A. Shaw, and D. G. Kay, "Panax ginseng is neuroprotective in a novel progressive model of Parkinson's disease," Experimental Gerontology, vol. 50, no. 1, pp. 95-105, 2014.

[79] J. Wu, H. K. Jeong, S. E. Bulin, S. W. Kwon, J. H. Park, and I. Bezprozvanny, "Ginsenosides protect striatal neurons in a cellular model of Huntington's disease," Journal of Neuroscience Research, vol. 87, no. 8, pp. 1904-1912, 2009.

[80] F. Jiang, S. DeSilva, and J. Turnbull, "Beneficial effect of ginseng root in SOD-1 (G93A) transgenic mice," Journal of the Neurological Sciences, vol. 180, no. 1-2, pp. 52-54, 2000.

[81] J. Hardy and D. Allsop, "Amyloid deposition as the central event in the aetiology of Alzheimer's disease," Trends in Pharmacological Sciences, vol. 12, pp. 383-388, 1991.

[82] K. Iqbal, A. del C Alonso, S. Chen et al., "Tau pathology in Alzheimer disease and other tauopathies," Biochimica et Biophysica Acta-Molecular Basis of Disease, vol. 1739, no. 2, pp. 198-210, 2005.

[83] X.-Y. Song, J.-F. Hu, S.-F. Chu et al., "Ginsenoside Rgl attenuates okadaic acid induced spatial memory impairment by the GSK3 $\beta$ /tau signaling pathway and the $\mathrm{A} \beta$ formation prevention in rats," European Journal of Pharmacology, vol. 710, no. 1-3, pp. 29-38, 2013.

[84] F.-C. Luo, S.-D. Wang, L. Qi, J.-Y. Song, T. Lv, and J. Bai, "Protective effect of panaxatriol saponins extracted from Panax notoginseng against MPTP-induced neurotoxicity in vivo," Journal of Ethnopharmacology, vol. 133, no. 2, pp. 448-453, 2011. 


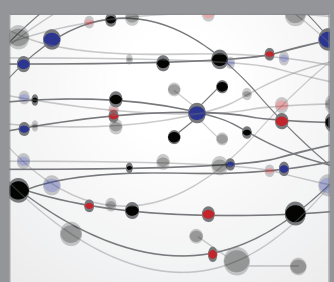

The Scientific World Journal
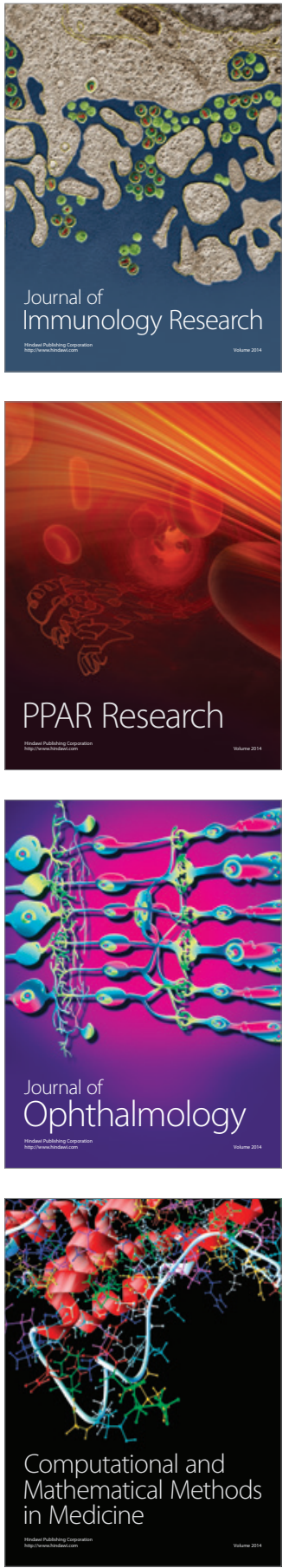

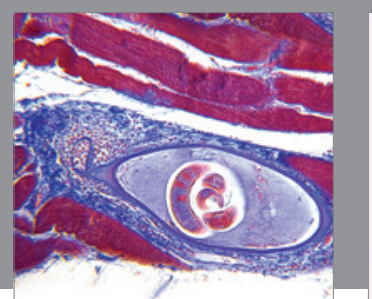

Gastroenterology Research and Practice

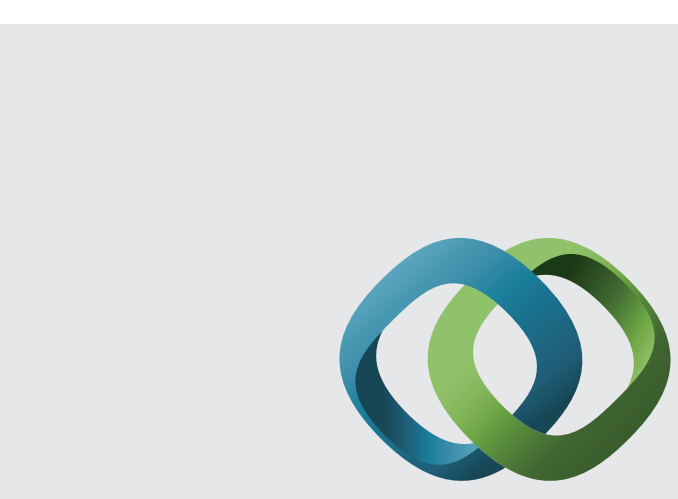

\section{Hindawi}

Submit your manuscripts at

http://www.hindawi.com
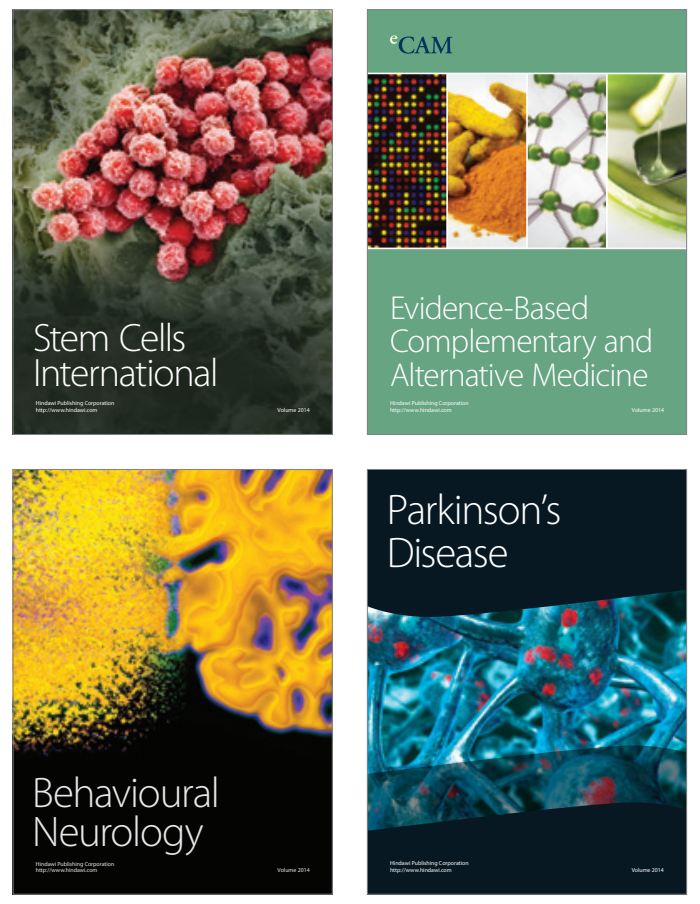
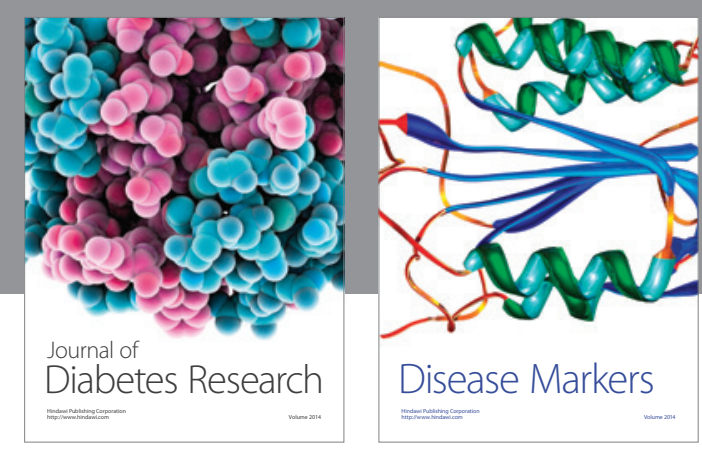

Disease Markers
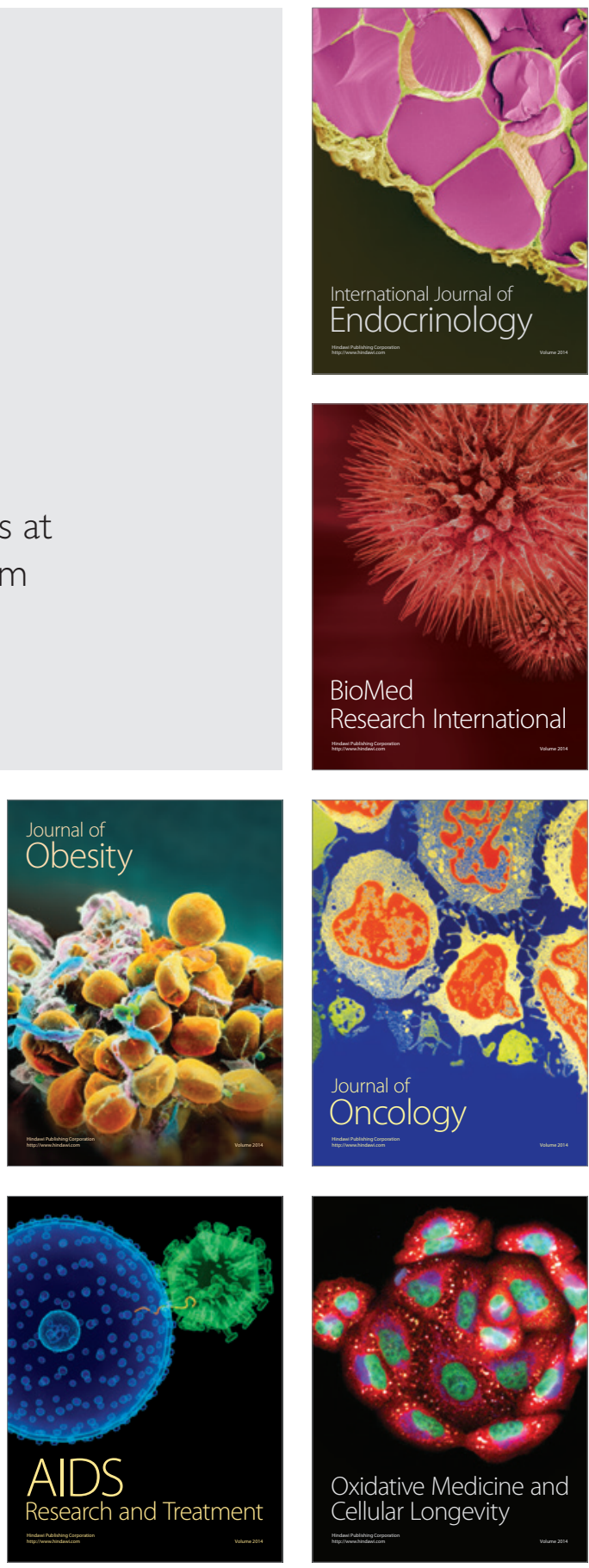